\title{
A Few TH-Immunoreactive Neurons Closely Appose DMX-Located Neuronal Somata Projecting to the Stomach Prepyloric Region in the Pig
}

\author{
Jaroslaw Calka ${ }^{1, *}$, Marta Ganko ${ }^{1}$ and Andrzej Rychlik ${ }^{2}$ (D) \\ 1 Department of Clinical Physiology, Faculty of Veterinary Medicine, University of Warmia and Mazury, \\ Oczapowskiego 13, 10-718 Olsztyn, Poland; marta.ganko@uwm.edu.pl \\ 2 Department of Clinical Diagnostics, Faculty of Veterinary Medicine, University of Warmia and Mazury, \\ Oczapowskiego 14, 10-718 Olsztyn, Poland; rychlik@uwm.edu.pl \\ * Correspondence: calkaj@uwm.edu.pl
}

Received: 23 October 2020; Accepted: 28 October 2020; Published: 31 October 2020

check for updates

Simple Summary: Although organization of the catecholaminergic system, in the porcine vagal motor nuclei of the pig, as well as distribution and chemical nature of the parasympathetic preganglionic neurons innervating the prepyloric region of the porcine stomach in the nucleus, have been well established, the question of a possible direct regulatory interaction between both neuronal systems still remains unknown. We discovered morphological foundations for direct regulatory action of the local TH-immunoreactive neurons on vagal preganglionic parasympathetic efferent neurons supplying the prepyloric region of the porcine stomach.

\begin{abstract}
The vagus nerve is responsible for efferent innervation and functional control of stomach functions. The efferent fibers originate from neurons located in the dorsal motor nucleus of the vagus (DMX) and undergo functional control of the local neuroregulatory terminals. The aim of the present study was to examine the existence of morphological foundations for direct regulatory action of the local TH-immunoreactive neurons on parasympathetic efferent neurons supplying the prepyloric region of the porcine stomach. Combined injection of neuronal retrograde tracer Fast Blue into the stomach prepyloric region with $\mathrm{TH}$ immunostaining was used in order to visualize spatial relationship between DMX-located stomach prepyloric region supplying neuronal stomata and local TH-IR terminals. We confirmed existence of TH-immunoreactive neural terminals closely opposing the stomach prepyloric region innervating neurons at the porcine DMX area. The observed spatial relationship points out the possibility of indirect catecholaminergic control of the stomach function exerted through preganglionic parasympathetic efferent neurons in the pig.
\end{abstract}

Keywords: dorsal motor vagal nucleus; stomach; prepyloric region; tyrosine hydroxylase

\section{Introduction}

It is generally accepted that the dorsal motor vagal nucleus (DMX) and the nucleus ambiguous constitute a source of preganglionic parasympathetic innervation for the upper gastrointestinal tract through many species, including the cat [1], dog [2], rat [3], as well as the domestic pig [4,5]. The authors' former tracing studies revealed that, in the pig, parasympathetic efferents supplying the stomach prepyloric region originated exclusively from the dorsal motor vagal nucleus [6], while the nucleus ambiguous innervated the esophagus [7].

The authors' recent immunocytochemical study confirmed the cholinergic nature of the vagal preganglionic perikarya and additionally revealed that their direct surroundings were comprised of numerous networks of nerve terminals possibly affecting their regulatory functions [8]. Thus, 
between the cholinergic somata, numerous substance P (SP-), leu5-enkephalin (LENK-), cocaine- and amphetamine-regulated transcript (CART-), and nitric oxide synthase (NOS-IR) protrusions were found, while SP- and LENK-IR processes formed a basket-like structure closely surrounding parasympathetic cholinergic neurons.

Consistently, others have found catecholaminergic neurons in rat [9], dog [10], sheep [11], human [12], and swine [13] DMX. Although in the latter, fluorogold tracing studies demonstrated the presence of catecholaminergic neurons intermingled between parasympathetic efferent somata in the DMX area, the question of the spatial relationship between porcine parasympathetic efferent neurons and catecholaminergic neurons remains unclear. Consequently, the problem of the possible presence of morphological foundations for the direct action of catecholaminergic neurons on preganglionic parasympathetic DMX somata supplying the stomach in the pig remains uncertain. The question of the spatial correlation has recently gained special attention, since DMX $\alpha_{2}$-adrenergic receptors are likely to mediate central gastroprotective action in the stomach [14]. Thus, based on the gathered data, it is hypothesized that, in the porcine DMX, there could be a spatial correlation between "gastric" parasympathetic somata and local adrenergic neurons.

Therefore, the main goal of this project was to study the possible existence of direct contact or direct opposition between the DMX-located catecholaminergic neurons and the retrogradely-traced DMX originating stomach prepyloric region innervating neurons in the pig. Moreover, possible co-expression of TH in those vagal "gastric" neurons was also investigated.

\section{Materials and Methods}

All procedures were conducted on five $(n=5)$ immature gilts (about $20 \mathrm{~kg}$ of body weight) of the Large White Polish breed kept in standard laboratory conditions with access to species-specific feed (Grower, Nutrena, Poland, metabolic energy 12.9 MJ) and tap water. All experimental procedures were performed in agreement with the rules approved by the Local Ethics Committee in Olsztyn (decision no. $05 / 2010)$.

Before administration (15 min) of the main anesthetic, sodium thiopental (Thiopental, Sandoz, Kundl-Rakusko, Austria) (10 mg/kg of body weight i.v.), the animals were pretreated with azaperone (Stresnil, Jansen Pharmaceutica N.V., Belgium) $(4 \mathrm{mg} / 1 \mathrm{~kg}$ of bodyweight, i.m.). The gilts were laparotomized to expose the stomach and a total volume of $50 \mu \mathrm{L}$ of $5 \%$ aqueous suspension of the retrograde neuronal tracer Fast Blue (FB, EMS-CHEMIE GmbH, Germany) was multiple injected ( $\mathrm{n}=50,1 \mu \mathrm{L}$ each) with a Hamilton syringe into the diamond-shaped part (ca. $4 \times 4 \mathrm{~cm}, \pm 16 \mathrm{~cm}^{2}$ ) of the diaphragmatic prepyloric area of the stomach (muscular and submucous layer) situated about $1 \mathrm{~cm}$ from the greater curvature of the stomach and $3 \mathrm{~cm}$ from the pylorus. To prevent leaking of the tracer outside the studied region, the needle was left in place for about $20 \mathrm{~s}$ after each injection.

Four weeks after FB injection, the gilts were euthanized by an overdose of sodium thiopental and transcardially perfused, with $4 \%$ buffered paraformaldehyde ( $\mathrm{pH} 7.4$ ).

Following perfusion, medulla oblongata blocks were collected from all animals, postfixed by immersion in the same fixative for $20 \mathrm{~min}$, rinsed with $0.1 \mathrm{M} \mathrm{PB}(\mathrm{pH} 7.4$ ) over three days, and then transferred to $30 \%$ buffered sucrose solution (pH 7.4), containing $0.01 \%$ natrium azide, and stored at $4{ }^{\circ} \mathrm{C}$. Finally, $14 \mu \mathrm{m}$ thick cryostat sections were prepared and mounted on chrome alum-coated slides (about 1000 sections from each medulla were cut in total, about 300 of them contained FB-labeled "gastric" neurons and were further stained for TH immunoreactivity), and then analyzed under an Olympus BX51 fluorescent microscope (Olympus, Tokyo, Japan) equipped with an appropriate filter for FB to localize the FB-labeled perikarya. To avoid double counting the same neuron, only the FB-labeled cell bodies with a visible nucleus in every fourth section were scored.

Selected sections of the medulla containing FB-positive perikarya were processed for double-labeling immunofluorescence staining. Briefly, after air-drying at room temperature for $45 \mathrm{~min}$ and washing in a $0.1 \mathrm{M}$ phosphate-buffered saline (PBS, pH 7.4, $3 \times 10 \mathrm{~min}$, the sections were incubated for $1 \mathrm{~h}$, in a blocking buffer containing 0.1\% BSA (bovine serum albumin) in $0.1 \mathrm{M}$ PBS, 
$1 \%$ Triton $\mathrm{X}-100,0.05 \%$ Thimerosal, $0.01 \%$ sodium azide, and again rinsed in PBS ( $3 \times 10 \mathrm{~min})$. Then, the sections were incubated overnight at room temperature with antisera (see Table 1), a mixture of ChAT (goat, AB144P-1ML, Millipore, Temecula, CA, USA, working dilution 1:50) and TH (mouse, MAB318, Millipore, Temecula, CA, USA, working dilution 1:200). On the following day, the sections were washed (PBS, $3 \times 10 \mathrm{~min}$ ) and incubated at room temperature, for $1 \mathrm{~h}$, with secondary antibodies, i.e., Alexa Fluor $488 \mathrm{~nm}$ anti-goat (A11055, Thermo Fisher Scientific, Waltham, MA, USA, working dilution 1:1000) and Alexa Fluor $546 \mathrm{~nm}$ anti-mouse (A10036, Thermo Fisher Scientific, Waltham, MA, USA working dilution 1:1000). After consequent rinsing in PBS $(3 \times 10 \mathrm{~min})$, the slides were coverslipped with carbonate-buffered glycerol ( $\mathrm{pH}$ 8.6).

Table 1. Description of antibodies.

\begin{tabular}{ccccc}
\hline Antigen & Species & Dilution & Code & Manufacturer/Supplier \\
\hline TH & Mouse & $1: 200$ & MAB318 & Millipore, Temecula, CA, USA \\
\hline ChAT & Goat & $1: 50$ & AB144P-1MLMillipore, Temecula, CA, USA \\
\hline Alexa Fluor 488 nm & anti-goat & 1:1000 & A11055 & $\begin{array}{c}\text { Thermo Fisher Scientific, } \\
\text { Waltham, MA, USA }\end{array}$ \\
\hline Alexa Fluor 546 nm & anti-mouse & 1:1000 & A10036 & $\begin{array}{c}\text { Thermo Fisher Scientific, } \\
\text { Waltham, MA, USA }\end{array}$ \\
\hline
\end{tabular}

The omission of primary antisera and their replacement by normal sera were applied to control the specificity of immunofluorescence. Staining was not observed in either case.

Finally, the slides were analyzed and photographed under an Olympus BX51 microscope equipped with epi-fluorescence and appropriate filter sets.

\section{Results}

The stomach projecting prikarya, labeled with FB, were found to be mostly oval or round in shape. Multipolar forms were occasionally encountered. Their nuclei were situated centrally and somata were measured as 20 to $50 \mu \mathrm{m}$ in diameter. They never co-expressed TH immunoreactivity, although all of them were ChAT-immunoreactive (Figure 1(1a-1c)).

Remarkably, among numerous FB-negative/ChAT-positive vagal perikarya, singular neurons were observed to express TH (Figure 1(1a-1c)). However, the remaining majority of the TH-IR neurons did not co-localize with ChAT. Catecholaminergic (FB-negative/ChAT-negative) perikarya were observed in the peripheral subregion of the DMX. The morphology of the majority of the TH-IR neurons varied from those of FB/ChAT-labeled cells. They were smaller in size (20 to $25 \mu \mathrm{m}$, occasionally $30 \mu \mathrm{m}$ in diameter) and often possessed fusiform cell bodies with long processes and unstained nuclei.

Most interestingly, very close direct apposition of those TH-IR cells with FB-positive/ChAT-labeled "prepyloric" neurons was encountered. Quantification revealed that $0.329 \%$ of the "prepyloric" neurons established apposition with TH-IR cells. They occurred peripherally in the dorsal and ventral subregion. The processes of the catecholaminergic somata wrapped around the FB-positive/ChAT-positive neuronal cell bodies, thus, establishing a very close spatial relationship between the opposing neurons (Figure 1(2a-2c)). 

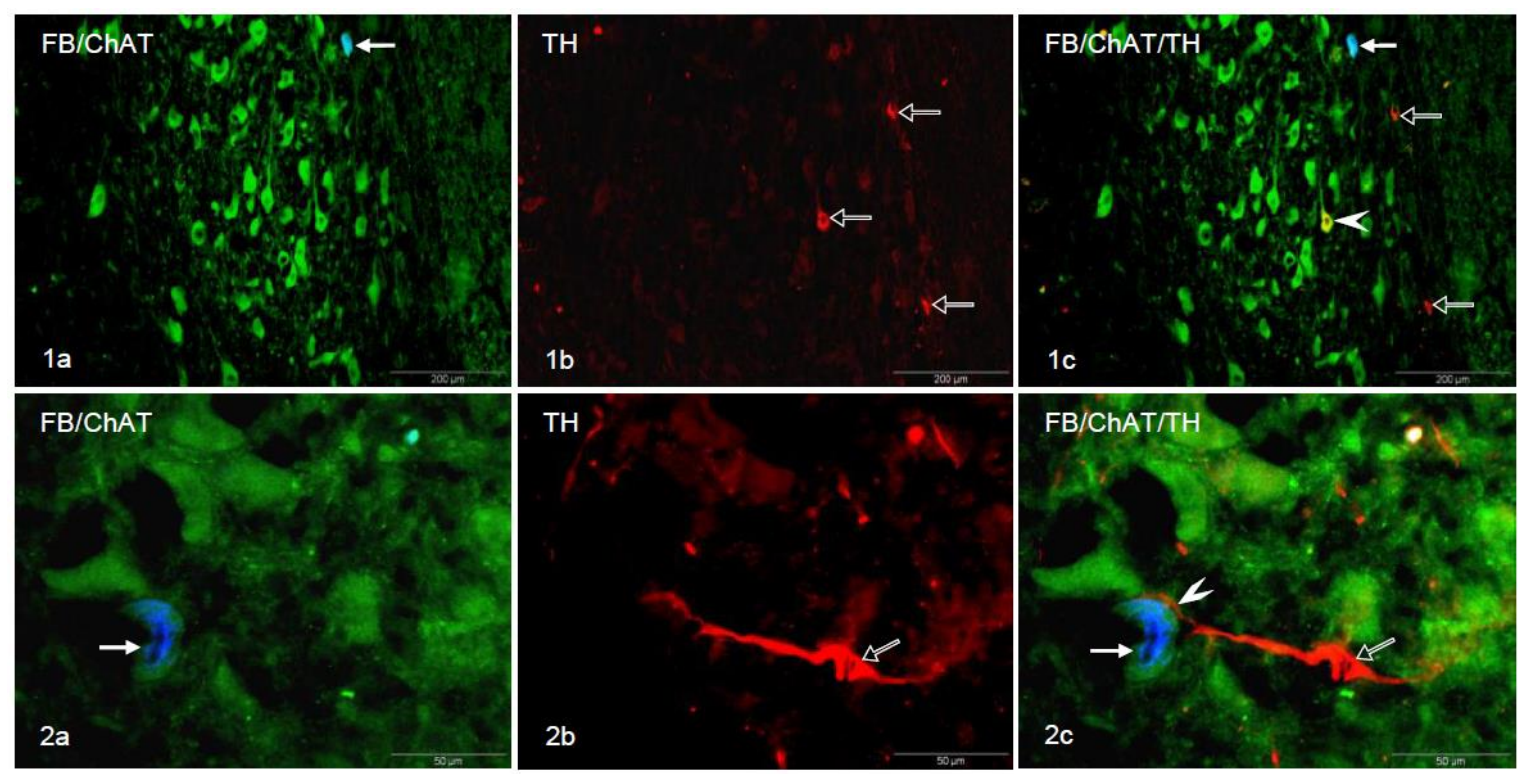

Figure 1. (1a) Double-labeled $\mathrm{FB}^{+} / \mathrm{ChAT}^{+}$neuron (bold arrow) in the porcine DMX; (1b) $\mathrm{TH}^{+}$neurons (empty arrows); (1c) Digital superimposition of pictures in (1a,1b). Double-labeled $\mathrm{FB}^{-} / \mathrm{ChAT}^{+} / \mathrm{TH}^{+}$ perikaryon (arrowhead), double-labeled $\mathrm{FB}^{+} / \mathrm{ChAT}^{+}$neuron (bold arrow) and $\mathrm{TH}^{+}$neurons (empty arrows) in the DMX. (2a) Double-labeled $\mathrm{FB}^{+} / \mathrm{ChAT}^{+}$perikaryon (arrow) in the porcine DMX; (2b) $\mathrm{TH}^{+}$ neuron (empty arrow); (2c) Digital superimposition of pictures in $(\mathbf{2 a}, \mathbf{2 b}) . \mathrm{TH}^{+}$neuron (empty arrow) in close proximity of the $\mathrm{FB}^{+} / \mathrm{ChAT}^{+}$perikaryon (arrow). The arrowhead indicates the place of direct opposition of the $\mathrm{TH}^{+}$process on the $\mathrm{FB}^{+} / \mathrm{ChAT}^{+}$neuronal soma.

\section{Discussion}

Combining injection of neuronal retrograde tracer Fast Blue into the stomach prepyloric region with TH immunostaining, the possibility of a close spatial relationship between vagal "prepyloric" motoneurons and TH-IR neurons in the porcine DMX region was demonstrated. Although Chaillou et al. [13] observed TH-IR neuronal, as well as vagal motor neuronal somata, in the porcine DMX, due to applied tracing procedure (transection of the left cervical vagal trunk), the authors were not able to specify the precise organ destination of the particular studied motoneuron. The selected approach allowed precise detection of the gastric "prepyloric" vagal motoneurons and their close spatial relationship with neighboring TH-immunoreactive neurons. The small number (see Table 2) of FB-labeled neurons apposing TH-IR terminals, found in the studied DMX, correlates with the limited surface area $\left( \pm 16 \mathrm{~cm}^{2}\right)$ of the stomach wall under study. Consequently, evidence was provided that in the porcine DMX, gastric "prepyloric" neurons most likely underwent direct functional control of the local catecholaminergic system. Moreover, we delivered evidence that those gastric "prepyloric" cholinergic motoneurons did not co-express TH. However, since individual cholinergic motoneurons of an unknown destination (FB-negative) have been found to express TH immunoreactivity, one could expect that, in the pig, as previously described by Tsukamoto et al. [15] in the rat, some abdominal organs, including other not studied stomach areas as well as thoracic organs, may receive DMX-originating singular cholinergic/catecholaminergic vagal efferents. Elucidation of this question needs additional tracing investigations. 
Table 2. Number of spatial appositions between $\mathrm{FB}^{+} / \mathrm{ChAT}^{+}$and $\mathrm{TH}^{+}$neurons.

\begin{tabular}{ccccccc}
\hline Pig & I & II & III & IV & V & $\begin{array}{c}\text { Mean } \pm \text { Standard } \\
\text { Error of Mean (SEM) }\end{array}$ \\
\hline $\begin{array}{c}\text { Number of appositions } \\
\text { between } \mathrm{CHAT}^{+} / \mathrm{FB}^{+} \text {and } \\
\mathrm{TH}^{+} \text {neurons }\end{array}$ & 1 & 2 & 0 & 2 & 3 & $\mathbf{1 . 6} \pm \mathbf{0 . 5}$ \\
\hline Number of $\mathrm{FB}^{+}$cells & 508 & 633 & 375 & 463 & 447 & $\mathbf{4 8 5 . 2} \pm \mathbf{4 2 . 7}$ \\
\hline
\end{tabular}

The current results, showing a close spatial relationship in the pig between vagal "prepyloric" somata and local TH-IR terminals in connection with a lack of TH-IR preganglionic neurons projecting directly to the prepyloric stomach region, corresponds with the results of Guo et al. [16] who found that, in the rat DMX, only $2 \%$ of the antrum projecting neurons expressed TH-IR. This interspecies similarity indicated that vagal DMX-located TH-IR neurons could indirectly, through preganglionic parasympathetic efferents, control stomach prepyloric functions. This hypothesis finds strong support in the results of Rosin et al. [17] and Tavares et al. [18], who reported abundant expression of $\alpha 2 \mathrm{~A}-$, $\alpha 2 \mathrm{~B}-$, and $\alpha 2 \mathrm{C}$-adrenergic receptor subtypes in neurons of the dorsal motor complex.

The presented results imply direct regulatory influence of the local DMX-located catecholaminergic neurons on the gastric vagal "preganglionic" somata. In the pig, the somata are also likely to be affected by other neuronal networks. Indeed, our former study [8] revealed networks of the SP-, LENK-, NOS and CART-immunoreactive processes located in close vicinity of the parasympathetic neurons. Nitrergic, as well as CART-IR terminals, formed basket-like structures closely surrounding DMX cholinergic neurons. Confirmation of such a functional relationship should come from the localization of the appropriate receptors on the vagal motoneurons.

The discovery of adjacent localization of the gastric vagal somata, in the current study, with nerve terminals originating from local DMX catecholaminergic neurons may constitute significant proof of the downregulatory effect of the vagal catecholaminergic neurons on the function of the vagal "prepyloric" parasympathetic projections. Nonetheless, further elucidation of this question, especially in the context of chemical adaptation of the neurons to catecholaminergic stimulation, requires additional studies.

\section{Conclusions}

The results of the current study may implicate direct regulatory action of the local catecholaminergic neurons on the stomach supplying vagal parasympathetic nerve cells in the dorsal motor vagal nucleus of the pig.

Author Contributions: Conceptualization, J.C.; investigation, J.C., M.G., A.R.; writing—original draft preparation, J.C.; writing-review and editing, J.C., M.G., A.R. All authors have read and agreed to the published version of the manuscript.

Funding: Supported by the Polish State Committee for Scientific Research no. 1890/B/P01/2010/39 and by the University of Warmia and Mazury in Olsztyn (statutory research) grant no. 15.610.003-300. Project financially co-supported by the Minister of Science and Higher Education in the range of the program entitled "Regional Initiative of Excellence" for the years 2019-2020, project no. 010/RID/2018/19, amount of funding 12.000.000 PLN.

Acknowledgments: The authors thank Andrzej Pobiedziński for his excellent technical assistance.

Conflicts of Interest: The authors declare no conflict of interests.

\section{References}

1. Kalia, M.; Mesulam, M.M. Brain stem projections of sensory and motor components of the vagus complex in the cat: I. The cervical vagus and nodose ganglion. J. Comp. Neurol. 1980, 193, 435-465. [CrossRef] [PubMed]

2. Chernicky, C.L.; Barnes, K.L.; Ferrario, C.M.; Conomy, J.P. Brainstem distribution of neurons with efferent projections in the cervical vagus of the dog. Brain Res. Bull. 1983, 10, 345-351. [CrossRef]

3. Scharoun, S.L.; Barone, F.C.; Wayner, M.J.; Jones, S.M. Vagal and gastric connections to the central nervous system determined by the transport of horseradish peroxidase. Brain Res. Bull. 1984, 13, 573-583. [CrossRef] 
4. Kitchell, R.L.; Stromberg, M.W.; Davis, L.H. Comparative study of the dorsal motor nucleus of the vagus nerve. Am. J. Vet. Res. 1977, 38, 37-49. [PubMed]

5. Hopkins, D.A.; Gootman, P.M.; Gootman, N.; Di Russo, S.M.; Zeballos, M.E. Brainstem cells of origin of the cervical vagus and cardiopulmonary nerves in the neonatal pig (Sus scrofa). Brain Res. 1984, 306, 63-72. [CrossRef]

6. Gańko, M.; Całka, J. Localization and chemical coding of the dorsal motor vagal nucleus (DMX) neurons projecting to the porcine stomach prepyloric area in the physiological state and after stomach partial resection. J. Mol. Neurosci. 2014, 52, 90-100. [CrossRef]

7. Wu, M.; Majewski, M.; Wojtkiewicz, J.; Vanderwinden, J.M.; Adriaensen, D.; Timmermans, J.P. Anatomical and neurochemical features of the extrinsic and intrinsic innervation of the striated muscle in the porcine esophagus: Evidence for regional and species differences. Cell Tiss. Res. 2000, 311, 289-297. [CrossRef] [PubMed]

8. Gańko, M.; Rychlik, A.; Całka, J. Immunohistochemical characterization of neurons and neuronal processes in the dorsal vagal nucleus of the pig. Pol. J. Vet. Sci. 2013, 16, 9-16. [CrossRef] [PubMed]

9. Armstrong, D.M.; Ross, C.A.; Pickel, V.M.; Joh, T.H.; Reis, D.J. Distribution of dopamine-, noradrenaline-, and adrenaline-containing cell bodies in the rat medulla oblongata: Demonstrated by the immunocytochemical localization of catecholamine biosynthetic enzymes. J. Comp. Neurol. 1982, 212, 173-187. [CrossRef] [PubMed]

10. Dormer, K.J.; Anwar, M.; Ashlock, S.R.; Ruggiero, D.A. Organization of presumptive catecholaminesynthesizing neurons in the canine medulla oblongata. Brain Res. 1993, 601, 41-64. [CrossRef]

11. Tillet, Y.; Thibault, J. Catecholamine-containing neurons in the sheep brainstem and diencephalon: Immunohistochemical study with tyrosine hydroxylase $(\mathrm{TH})$ and dopamine-beta-hydroxylase $(\mathrm{DBH})$ antibodies. J. Comp. Neurol. 1989, 290, 69-104. [CrossRef] [PubMed]

12. Halliday, G.M.; Li, Y.W.; Joh, T.H.; Cotton, R.G.; Howe, P.R.; Geffen, L.B.; Blessing, W.W. Distribution of monoamine-synthesizing neurons in the human medulla oblongata. J. Comp. Neurol. 1988, 273, 301-317. [CrossRef] [PubMed]

13. Chaillou, E.; Tillet, Y.; Malbert, C.H. Organisation of the catecholaminergic system in the vagal motor nuclei of pigs: A retrograde fluorogold tract tracing study combined with immunohistochemistry of catecholaminergic synthesizing enzymes. J. Chem. Neuroanat. 2009, 38, 257-265. [CrossRef] [PubMed]

14. Gyires, K.; Toth, V.E.; Zadori, Z.S. Gastric mucosal protection: From the periphery to the central nervous system. J. Physiol. Pharmacol. 2015, 66, 319-329. [PubMed]

15. Tsukamoto, K.; Hayakawa, T.; Maeda, S.; Tanaka, K.; Seki, M.; Yamamura, T. Projections to the alimentary canal from the dopaminergic neurons in the dorsal motor nucleus of the vagus of the rat. Auton. Neurosci. 2005, 123, 12-18. [CrossRef]

16. Guo, J.J.; Browning, K.N.; Rogers, R.C.; Travagli, R.A. Catecholaminergic neurons in rat dorsal motor nucleus of vagus project selectively to gastric corpus. Am. J. Physiol. Gastrointest. Liver Physiol. 2001, 280, G361-G367.

17. Rosin, D.L.; Talley, E.M.; Lee, A.; Stornetta, R.L.; Gaylinn, B.D.; Guyenet, P.G.; Lynch, K.R. Distribution of alpha-2C -Adrenergic Receptor-Like Immunoreactivity in the Rat Central Nervous System. J. Comp. Neurol. 1996, 372, 135-165. [CrossRef]

18. Tavares, A.; Handy, D.E.; Bogdanova, N.N.; Rosene, D.L.; Gavras, H. Localization of $\alpha 2 \mathrm{~A}$ and $\alpha 2 \mathrm{~B}-$ Adrenergic Receptor Subtypes in Brain. Hypertension 1996, 27, 449-455. [CrossRef] [PubMed]

Publisher's Note: MDPI stays neutral with regard to jurisdictional claims in published maps and institutional affiliations.

(C) 2020 by the authors. Licensee MDPI, Basel, Switzerland. This article is an open access article distributed under the terms and conditions of the Creative Commons Attribution (CC BY) license (http://creativecommons.org/licenses/by/4.0/). 